\title{
Patients with isolated vertigo attack preceding stroke have different clinic characteristics: a retrospective study
}

Dongxu Qiu ( $\sim 1007351969 @ q q . c o m$ )

Center South University

Lei Zhang

Center South Univetsity

Jun Deng

Center South University

Zhiwei Xia

Center South University

Jingfeng Deng

The Third Hospital of Changsha

Research article

Keywords: Vertigo attack, cerebral infarction, hemiplegia, vertebrobasilar stroke

Posted Date: June 26th, 2020

DOl: https://doi.org/10.21203/rs.3.rs-36693/v1

License: (9) This work is licensed under a Creative Commons Attribution 4.0 International License. Read Full License 


\section{Abstract \\ Background}

Isolated vertigo attack history preceding the acute stroke were frequently accompanying with other focal neurological symptoms. To clarify the different clinical characteristics between isolated vertigo attack and vertigo symptom accompanying hemiplegia preceding stroke, we performed this 4-year retrospective study.

\section{Methods}

Medical records of 1283 patients hospitalized with vertigo symptom had been screened. Patients were divided into two groups: isolated vertigo attack history preceding the stroke defined as IVA group, vertigo symptom accompanying hemiplegia attack defined as VAH group. Clinic characteristics including $A B C D^{2}$ score, infarction volume and location, relative risk factors and the following medical intervention were compared between the group.

\section{Results}

Patients featured with VAH had higher extracranial stenosis $(21.2 \%$ vs. $9.0 \%, \mathrm{P}<0.01)$ and $A B C D^{2}$ score ( $3.7 \pm 1.9$ vs. $2.3 \pm 1.5, P=0.03)$, patient with IVA showed a higher diabetic prevalence $(40.9 \%$ vs. $29.7 \%, P$ $=0.02$ ). The frequency of vertigo events tended to be more commonly in patient with VAH (median 3.1 vs. $5.5, p<0.03$ ). The total cerebral infarction volume in IVA group tended to be larger than VAH with a median of $4.56 \mathrm{~cm}^{3}$ versus $2.32 \mathrm{~cm}^{3}(p=0.02)$. Additionally, less patients with IVA sought medical intervention when vertigo symptom occurred.

\section{Conclusions}

Clinical characteristics including ABCD2 score, total cerebral infarction volume and the location were different between AVH and IVH group. In addition, less patients in IVH cohort sought medical intervention when vertigo symptom occurred.

\section{Background}

In recent years, cerebrovascular disease as a cause of isolated vertigo had been gained increased attention. However, due to the absence of highly sensitive diagnostic tools, the ability to clinically ascribe transient vertigo symptom to ischemic attack remains limited[1,2]. This holds particularly true when the atypical symptom presented with normal neurologic examination findings[3] $]^{3}$. Therefore, recurrent vertigo attack always misdiagnosed as migraine or Ménierè's disease rather than ischemic events. In addition, it 
could be more or less objective if the diagnosis depends on clinical experience other than on neurological imaging $[4,5]$. This situation happens frequency on junior physician at their first contact of isolated vertigo symptom cases[6]. Unfortunately, failure recognizing the vertigo attack timely may result in worse outcomes due to missing opportunity for acute stroke therapies [7].

Isolated vertigo is a common presentation among patients examined by neurologists, which comprises $47-75 \%$ of patients with posterior-circulation stroke $[8,9]$. But in some cases, patients have more than the single vertigo attack symptom at a time when they consult a physician[10]. For example, it can be accompanied by sudden numbness or weakness in face arm or soft palate when it involved with lateral part of the brain stem[11]. In addition, those atypical neurologic symptoms may transition from one to another over time. For these reasons, it is important to clarify the clinical characteristics between single vertigo attack and vertigo accompanying hemiplegia preceding stroke. However, no reliable data had been published to verify this issue. To address the question, we conducted a retrospective analysis between VAH and IVA cohort preceding acute vertebrobasilar (VB) stroke. The aims of the present study were to determine (i) clinic characteristics including $A B C D^{2}$ score, infarction volume and location between VAH and IVA patients (ii) high risk factors and immediately medical intervention after VAH/IVA occurred.

\section{Material}

\section{Study subjects}

Data for this retrospective study was collected from the patients with vertigo symptom as the main complaint in Xiangya Hospital and the Third Hospital of Changsha from 2014 to 2018. The study was approved by the ethics committee of Center South University. Each patient in the study signed the informed consent document.

\section{Definition of VB stroke}

During the hospitalization, all the participants involved the study enquired by the specially trained neurologist about whether they had hemiplegia attack history within 3 months preceding VB stroke. Hemiplegia attack were defined as numbness or weakness in face, arm or soft palate. Similar questions were enquired for vertigo attack history. Vertigo symptom was defined according to the criteria of Bárány Society $[12,13]$. If the patients responded affirmatively, additional questions regarding the exact time of onset, detailed description of symptom, frequency and duration of attack, and whether they received the medical intervention were asked. VB stroke was determined as an episode of acute focal neurologic deficits with symptoms lasting more than 24 hours. In addition, acute cerebral infarction corresponding to the current neurological deficits was supported by magnetic resonance imaging (MRI). All the acute VB stroke were diagnosed by trained neurologists based on clinical characteristics and MRI evidence or CT findings.

\section{Definition of vascular risk factors and infarction volume}


The following vascular risk factors were analyzed for the purpose of this study: diabetes mellitus (previous diagnosis of diabetes mellitus or take the hypoglycemic agents currently), hypertension (diastolic blood pressure $>90 \mathrm{mmHg}$ and/or systolic $>140 \mathrm{mmHg}$, or take the antihypertensive medications currently), hyperlipidemia (triglycerides $>1.71 \mathrm{mmol} / \mathrm{L}$ and/or cholesterol $>5.17 \mathrm{mmol} / \mathrm{L}$ ), alcohol consumption during the past 3 month (the standard alcohol consumption criteria is equivalent to $300 \mathrm{~mL}$ of beer or $100 \mathrm{~mL}$ of wine), smoking (continuous or cumulative history of smoking $>9$ month and $\geq 1$ cigarette per day)[14]. Carotid and vertebral artery ultrasound were detected by specially trained neurologists. The diagnosis of extracranial arterial stenosis was defined as artery stenosis $>50 \%[15]$. MRI scan was performed on a GE Signa HDX 3.0T MRI (Fairfield, USA). The acquisition sequence of T1 and T2-weighted scans, Diffusion-weighted imaging (DWI), apparent on diffusion coefficient (ADC) and fluid attenuated inversion recovery (FLAIR) imaging were obtained per patient. DWI data were acquired by using fast spin-echo planar imaging sequence with TE 77.6, TR 50000, slice thickness of $5.0 \mathrm{~mm}$ and field of view $220 \times 220 \mathrm{~mm}$. Maximum-intensity projection of a three-dimensional volume was applied in data acquisition and imaging reconstruction. All the procedures involved this study were performed on a post-processing GE machine (Siemens, Inc., Munich, Germany). The detailed information for further analysis were obtained by two experienced observers to reduce the risk of unconscious bias.

\section{Statistical Analysis}

Statistical analyses of this study were performed by using SPSS software (version 23.0; IBM, Chicago, IL, USA). Continuous variables with normal distribution were expressed as mean \pm standard deviation. The unpaired two-samples t-test was used for two independent group comparison. The one-way analysis of variance (ANOVA) was used to compare the differences for three or more groups. if not normally distributed data, nonparametric tests are performed and were presented as median (range). MannWhitney U-test were used to compare the difference between the groups, P value $<0.05$ is considered to be statistically significant.

\section{Results}

We screened medical records of 1283 consecutive patients hospitalized with vertigo as the main complaint. Overall, 437 patients had final diagnosis of Ménierè's disease $(n=132)$, migraine $(n=106)$, orthostatic hypotension $(n=68)$, Coronary heart disease $(n=66)$ and psychiatric disorders $(n=65)$. All of them were excluded from the analysis. The remaining 350/846 (41.3\%) and 496/846 (58.6\%) patients had a definite ischemic diagnosis as transient ischemic attacks (TIAs) or stroke. Among of those stroke cases, $360(72.5 \%)$ involved in vertebrobasilar (VB) territory and $136(27.4 \%)$ in carotid territory. Of those VB stroke cases, 66 had recurrent isolated vertigo attack history preceding the stroke (IVA), 94 had vertigo symptom accompanying hemiplegia attack history (VAH). Detailed information was presented in study flow chart (Fig. 1).

In a comparison of vascular risk factors between IVA and VAH group, patients with VAH had higher extracranial stenosis $(21.2 \%$ vs. $9.0 \%, P<0.01)$ and $A B C D^{2}$ score $(3.7 \pm 1.9$ vs. $2.3 \pm 1.5, P=0.03)$. 
However, patients with IVA showed a higher prevalence of diabetic mellitus $(40.9 \%$ vs. $29.7 \%, P=0.02)$. Other atherothrombotic risk factors, including current smoking $(21.8 \%$ vs. $26.5 \%)$, history of hyperlipidemia (31.8\% vs. $34.0 \%$ ), hypertension ( $54.5 \%$ vs. $48.9 \%$ ), alcohol ( $15.1 \%$ vs. $13.8 \%)$, and peripheral vascular disease (6.0\% vs. $8.5 \%$ ) were not differ significantly between the group (Table 1$)$.

Table 1

Patient demographics and baseline characteristics $(n=160)$

\begin{tabular}{|c|c|c|c|}
\hline & IVH $(n=66)$ & $\operatorname{VAH}(n=94)$ & $P$-Value \\
\hline Male $(n, \%)$ & $30(45.4 \%)$ & $53(56.3 \%)$ & 0.25 \\
\hline Age, Mean \pm SD (years) & $65.3 \pm 10.5$ & $62.6 \pm 11.6$ & 0.32 \\
\hline Hypertension (n, \%) & $36(54.5 \%)$ & $46(48.9 \%)$ & 0.12 \\
\hline Diabetes mellitus (n, \%) & $27(40.9 \%)$ & $28(29.7 \%)$ & $0.02^{*}$ \\
\hline Body mass index, $\mathrm{kg} / \mathrm{m} 2$ & $25.9 \pm 5.4$ & $25.1 \pm 4.9$ & 0.31 \\
\hline Coronary heart disease $(n, \%)$ & $6(9.0 \%)$ & $11(11.7 \%)$ & 0.54 \\
\hline Extracranial stenosis $(\mathrm{n}, \%)$ & $6(9.0 \%)$ & $20(21.2 \%)$ & $<0.01^{*}$ \\
\hline peripheral vascular disease $(n, \%)$ & $4(6.0 \%)$ & $8(8.5 \%)$ & 0.67 \\
\hline Dyslipidemia (n, \%) & $21(31.8 \%)$ & $32(34.0 \%)$ & 0.78 \\
\hline Current smoking (n, \%) & $14(21.8 \%)$ & $25(26.5 \%)$ & 0.43 \\
\hline Alcoholism (n, \%) & $10(15.1 \%)$ & $13(13.8 \%)$ & 0.40 \\
\hline$A B C D^{2}$ score, Mean $\pm S D$ & $2.3 \pm 1.5$ & $3.7 \pm 1.9$ & $0.03^{*}$ \\
\hline Score $\geq 4(n, \%)$ & $19(28.7 \%)$ & $45(47.8 \%)$ & $0.02^{*}$ \\
\hline \multicolumn{4}{|c|}{$\begin{array}{l}\text { IVH: recurrent isolated vertigo attack history preceding the stroke; } \mathrm{AVH} \text { : vertigo symptom } \\
\text { accompanying numbness or weakness attack in face, arm or soft palate history preceding the stroke; } \\
\text { * Statistically significant, } \mathrm{P}<0.05\end{array}$} \\
\hline
\end{tabular}

Comparisons were made regarding to the vertigo events occurring 30 days prior to the acute VB stroke. It showed that the episodes of vertigo attack tended to be more frequent in patients with VAH (median 3.1 vs. $5.5, \mathrm{p}<0.03$ ) (Fig. 2A). In addition, although the mean interval between the first vertigo symptom occurred and subsequent VB stroke (median 6.9 vs. 8.1 days, $P=0.07$ ) showed no differences (Fig. 2B), 53 patients $(56.3 \%, 53 / 94)$ of VAH cohort sought medical attention after vertigo arise, which showed much less in IVA cohort (36.3\%, 24/66, Fig. 2C).

Cerebral infarction location was analysis between IVA and VAH further. The posterior cerebellum was more frequently involved in IVA group $(68.1 \%$ vs. $27.6 \%, p<0.001)$. However, VAH group are more likely to be involved in lateral medullary ( $38.2 \%$ vs. $12.1 \% ; \mathrm{P}=0.03)($ Table 2$)$. In addition, the total infarction 
volume in IVA tended to be larger than VAH with a median of $4.56 \mathrm{~cm}^{3}$ versus $2.32 \mathrm{~cm}^{3}(p=0.02, F i g .2 D)$. This difference was even more noticeable for territorial infarction located in posterior cerebellum with a median of 4.84 versus $1.78 \mathrm{~cm}^{3}$. By using a receiver operating characteristic (ROC) curve, a cutoff volume of $>3.99 \mathrm{~cm}^{3}$ for infarction located in the posterior cerebellum was found to be determine IVA from VAH with specificity of $76.2 \%$ and sensitivity of $73.4 \%$; $A U C(95 \% \mathrm{Cl})=0.718(0.615,0.820), p=0.002$ (Fig. 3).

Table 2

Analysis of infarction location

\begin{tabular}{|c|c|c|c|}
\hline Infarction location & IVH (n, \%) & $\operatorname{VAH}(n, \%)$ & $P$-Value \\
\hline Anterior cerebellum & $36(54.5 \%)$ & $34(36.1 \%)$ & 0.61 \\
\hline Posterior cerebellum & $45(68.1 \%)$ & $26(27.6 \%)$ & $<0.001^{*}$ \\
\hline Medial medullary & $12(18.1 \%)$ & $25(26.5 \%)$ & 0.07 \\
\hline Lateral medullary & $8(12.1 \%)$ & $36(38.2 \%)$ & $0.03^{*}$ \\
\hline Anteromedial pontine & $5(7.5 \%)$ & $11(11.7 \%)$ & 0.41 \\
\hline Anterolateral pontine & $6(9.0 \%)$ & $15(15.9 \%)$ & 0.54 \\
\hline Dorsolateral pontine & $9(13.6 \%)$ & $12(12.7 \%)$ & 0.79 \\
\hline Midbrain infarction & $12(18.1 \%)$ & $10(10.6 \%)$ & 0.50 \\
\hline
\end{tabular}

\section{Discussion}

Subsequent VB stroke always be a concern in patients that presented with recurrent isolated vertigo symptom. Of note, isolated vertigo attack history preceding the VB stroke were frequently accompanying with other focal neurological symptoms[16, 17]. Although acute cerebral infarction causes focal neurological depicts suddenly and the predictable parametric causal factors are still lacking. However, most VB stroke patients reported vertigo attack had other preceding symptoms. This holds particularly true for isolated vertigo accompanying hemiplegia prior stroke. By screening all medical records of 1283 consecutive patients hospitalized with vertigo as the main complaint, 160 of them validated to have acute VB stroke. We showed that patients with IVA were different both in high risk factors and infarction location compared to VAH cohort. To our knowledge, no prior studies exist in literatures that focused on comparing isolated vertigo symptom and vertigo overlapping with hemiplegia attack preceding the VB stroke.

The spectrum of signs and symptoms associated with isolated vertigo attack preceding stroke depended on the affected vascular territories[18]. However, the situation could be highly variable and more complex 
when vertigo accompany with hemiplegia attack symptoms. By comparing the VAH and IVA clinic characteristics, we showed that the episode of vertigo events tended to be more frequent in patients with VAH. Although no statistically differences were found in the final incidence of acute stroke rate, patients with IVA were more likely to be involved in vascular territories such as posterior cerebellum. Since the brain territory of cerebellum was mainly perfused by posterior-inferior cerebellar artery (PICA)[19], it is important to note that recurrent isolated vertigo attack could be the sole sign and symptom preceding VB stroke when it involved with PICA artery. Conversely, the location of infarction involved in VAH patients were quite different from IVA as lateral medulla region had higher risky to be affected. Patients commonly manifested by nausea, vertigo along with unilateral numbness or weakness of the face or soft palate, those symptoms were in consistent with the transient attack history prior VB stroke in VAH cohort. In addition, we found that patients with $\mathrm{VAH}$ had higher $A B C D^{2}$ score and larger cerebrum infarction volume. Recurrent vertigo attack can herald an acute infarction event, and the $A B C D^{2}$ score is a clinical prediction of stroke by distinguishing TIA from mimics[20, 21]. We wonder whether this kind of higher $A B C D^{2}$ score is correlated to the larger infarction volume outcome in patient with VAH. However, little study has been found to illustrate the association between $A B C D^{2}$ score and the infarction volume in VB stroke. Further studies with larger cohorts are required to confirm this finding.

Recurrent vertigo attack providing a critical opportunity to identify and prevent a potentially devastating stroke[22, 23]. Without timely intervention, the relatively risk of stroke is as high as $20 \%$ over the next 3 months[24]. Within our cohort, even though the mean interval between the first vertigo event and the subsequent stroke were almost equivalent between the group. Regretfully, less patients in IVA cohort sought medical intervention after vertigo symptom arise. It is possible that patients presenting with vertigo accompanying hemiplegia attack tended to be more intense or impressive, persuading them to make an urgent clinic appointment. In addition, patients with VAH showed a higher prevalence of extracranial stenosis, which reminded them to seek for the most appropriate treatment to the primary physician. Timely treatment following isolated vertigo attack presents an opportunity to reduce the stroke burden in those patients. However, it is difficult to perform the whole test battery and imaging scan for patients with IVA immediately due to the poor help-seeking behavior. Previously data indicated that immediate clinical diagnosis following prompt medical intervention associated with a reduction as much as $80 \%$ in the risk of subsequent stroke[25,26]. Thus, timely treatment following vertigo attack presents an opportunity to reduce the stroke burden in those patients. However, it remains a challenge to perform the bedside examination and routine MRIs scan for patient with IVA timely due to their poor medical consultant behavior.

Our findings have implications to improve the public recognition and awareness between IVA and VAH attack. However, there are limitations of this study. The non-randomized recruitment of patients should be kept in mind before a conclusion drawn from the study. Second, as patient with mild stroke were often unwilling to visit the hospital when the neurological symptom resolved spontaneously. Therefore, the proportion of stroke may have been underestimated in this regard. It also should be acknowledged that the relatively small sample size might have resulted in a selection bias. 


\section{Conclusion}

We found that the episodes of vertigo events tended to be more frequent in VAH patients. Infarction located in lateral medullary was significantly more common in VAH patients. However, IVH patients are more likely to be involved in posterior cerebellum. In addition, the total infarction volume in IVH patients tended to be larger than VAH group. More importantly, even though the mean interval between the first vertigo event and the subsequent stroke were almost equivalent, less patients in IVH cohort sought medical intervention after vertigo symptom arise.

\section{Abbreviations}

VB: vertebrobasilar, IVA: isolated vertigo attack history preceding the stroke, VAH:vertigo symptom accompanying hemiplegia attack, TIAs: transient ischemic attacks

\section{Declarations}

\section{Acknowledgements}

We sincerely thank the cooperation of patients and their families in Xiangya Hospital and the Third Hospital of Changsha. The authors will share their research data, including, but not limited to, raw data, processed data, software, methods and materials.

\section{Author contributions}

DX drafting the manuscript; JF designed the study, statistical analysis, study supervision. ZW, JD, LZ, critical revision of the manuscript for intellectual content. JF final revise the manuscript.

\section{Funding}

This work was supported by grants from National Natural Science Foundation of China (No. 81974223)

\section{Availability of data and materials}

The datasets used and analysed during the current study are available from the corresponding author on reasonable request.

\section{Consent for publication}

Not applicable

\section{Competing interests}

The authors declare that the research was conducted in the absence of any commercial or financial relationships that could be construed as a potential conflict of interest. 


\section{Author details}

${ }^{1}$ Department of Neurology, Xiangya Hospital, Central South University, Changsha, PR China

${ }^{2}$ Department of Geriatrics, The third hospital of Changsha, Hunan 410011, PR China

\section{References}

1. Kraaijeveld CL, van Gijn J, Schouten HJ, Staal A. Interobserver agreement for the diagnosis of transient ischemic attacks. Stroke. 1984;15(4):723-5.

2. Tarnutzer AA, Berkowitz AL, Robinson KA, Hsieh YH, Newman-Toker DE. Does my dizzy patient have a stroke? A systematic review of bedside diagnosis in acute vestibular syndrome. CMAJ 2011, 183(9):E571-592.

3. Smith HE, Rynning RE, Okafor C, Zaslavsky J, Tracy JI, Ratliff J, et al. Evaluation of neurologic deficit without apparent cause: the importance of a multidisciplinary approach. J Spinal Cord Med. 2007;30(5):509-17.

4. Lee H. Isolated vascular vertigo. J Stroke. 2014;16(3):124-30.

5. Wintermark M, Sanelli PC, Albers GW, Bello J, Derdeyn C, Hetts SW, et al. Imaging recommendations for acute stroke and transient ischemic attack patients: A joint statement by the American Society of Neuroradiology, the American College of Radiology, and the Society of Neurolnterventional Surgery. AJNR Am J Neuroradiol. 2013;34(11):E117-27.

6. Nadarajan V, Perry RJ, Johnson J, Werring DJ. Transient ischaemic attacks: mimics and chameleons. Pract Neurol. 2014;14(1):23-31.

7. von Brevern M, Radtke A, Lezius F, Feldmann M, Ziese T, Lempert T, et al. Epidemiology of benign paroxysmal positional vertigo: a population based study. J Neurol Neurosurg Psychiatry. 2007;78(7):710-5.

8. Akhtar N, Kamran SI, Deleu D, D'Souza A, Miyares F, Elsotouhy A, et al. Ischaemic posterior circulation stroke in State of Qatar. Eur J Neurol. 2009;16(9):1004-9.

9. Choi JH, Kim HW, Choi KD, Kim MJ, Choi YR, Cho HJ, et al. Isolated vestibular syndrome in posterior circulation stroke: Frequency and involved structures. Neurol Clin Pract. 2014;4(5):410-8.

10. Lee JO, Park SH, Kim HJ, Kim MS, Park BR, Kim JS. Vulnerability of the vestibular organs to transient ischemia: implications for isolated vascular vertigo. Neurosci Lett. 2014;558:180-5.

11. Benghanem S, Mazeraud A, Azabou E, Chhor V, Shinotsuka CR, Claassen J, et al. Brainstem dysfunction in critically ill patients. Crit Care. 2020;24(1):5.

12. Bisdorff A, Von Brevern M, Lempert T, Newman-Toker DE. Classification of vestibular symptoms: towards an international classification of vestibular disorders. J Vestib Res. 2009;19(1-2):1-13.

13. Yao Q, Wang H, Song Q, Shi H, Yu D. Use of the Barany Society criteria to diagnose benign paroxysmal positional vertigo. J Vestib Res. 2018;28(5-6):379-84. 
14. Zhang DP, Li HR, Ma QK, Yin S, Peng YF, Zhang HL, et al. Prevalence of Stroke and Hypoperfusion in Patients With Isolated Vertigo and Vascular Risk Factors. Front Neurol. 2018;9:974.

15. Mokin M, Dumont TM, Kass-Hout T, Levy El. Carotid and vertebral artery disease. Prim Care. 2013;40(1):135-51.

16. Blasberg TF, Wolf L, Henke C, Lorenz MW. Isolated transient vertigo: posterior circulation ischemia or benign origin? BMC Neurol. 2017;17(1):111.

17. Kerber KA, Brown DL, Lisabeth LD, Smith MA, Morgenstern LB. Stroke among patients with dizziness, vertigo, and imbalance in the emergency department: a population-based study. Stroke. 2006;37(10):2484-7.

18. Choi KD, Lee H, Kim JS. Ischemic syndromes causing dizziness and vertigo. Handb Clin Neurol. 2016;137:317-40.

19. Chandra A, Li WA, Stone CR, Geng X, Ding Y. The cerebral circulation and cerebrovascular disease I: Anatomy. Brain Circ. 2017;3(2):45-56.

20. Navi BB, Kamel H, Shah MP, Grossman AW, Wong C, Poisson SN, et al. Application of the ABCD2 score to identify cerebrovascular causes of dizziness in the emergency department. Stroke. 2012;43(6):1484-9.

21. Sheehan OC, Merwick A, Kelly LA, Hannon N, Marnane M, Kyne L, et al: Diagnostic usefulness of the ABCD2 score to distinguish transient ischemic attack and minor ischemic stroke from noncerebrovascular events: the North Dublin TIA Study. Stroke 2009, 40(11):3449-3454.

22. Markus HS, van der Worp HB, Rothwell PM. Posterior circulation ischaemic stroke and transient ischaemic attack: diagnosis, investigation, and secondary prevention. Lancet Neurol. 2013;12(10):989-98.

23. Rothwell PM, Giles MF, Chandratheva A, Marquardt L, Geraghty O, Redgrave JN, et al. Effect of urgent treatment of transient ischaemic attack and minor stroke on early recurrent stroke (EXPRESS study): a prospective population-based sequential comparison. Lancet. 2007;370(9596):1432-42.

24. Khare S. Risk factors of transient ischemic attack: An overview. J Midlife Health. 2016;7(1):2-7.

25. Newman-Toker DE, Cannon LM, Stofferahn ME, Rothman RE, Hsieh YH, Zee DS. Imprecision in patient reports of dizziness symptom quality: a cross-sectional study conducted in an acute care setting. Mayo Clin Proc. 2007;82(11):1329-40.

26. Rothwell PM, Algra A, Chen Z, Diener HC, Norrving B, Mehta Z. Effects of aspirin on risk and severity of early recurrent stroke after transient ischaemic attack and ischaemic stroke: time-course analysis of randomised trials. Lancet. 2016;388(10042):365-75.

\section{Figures}




\section{ROC curve}

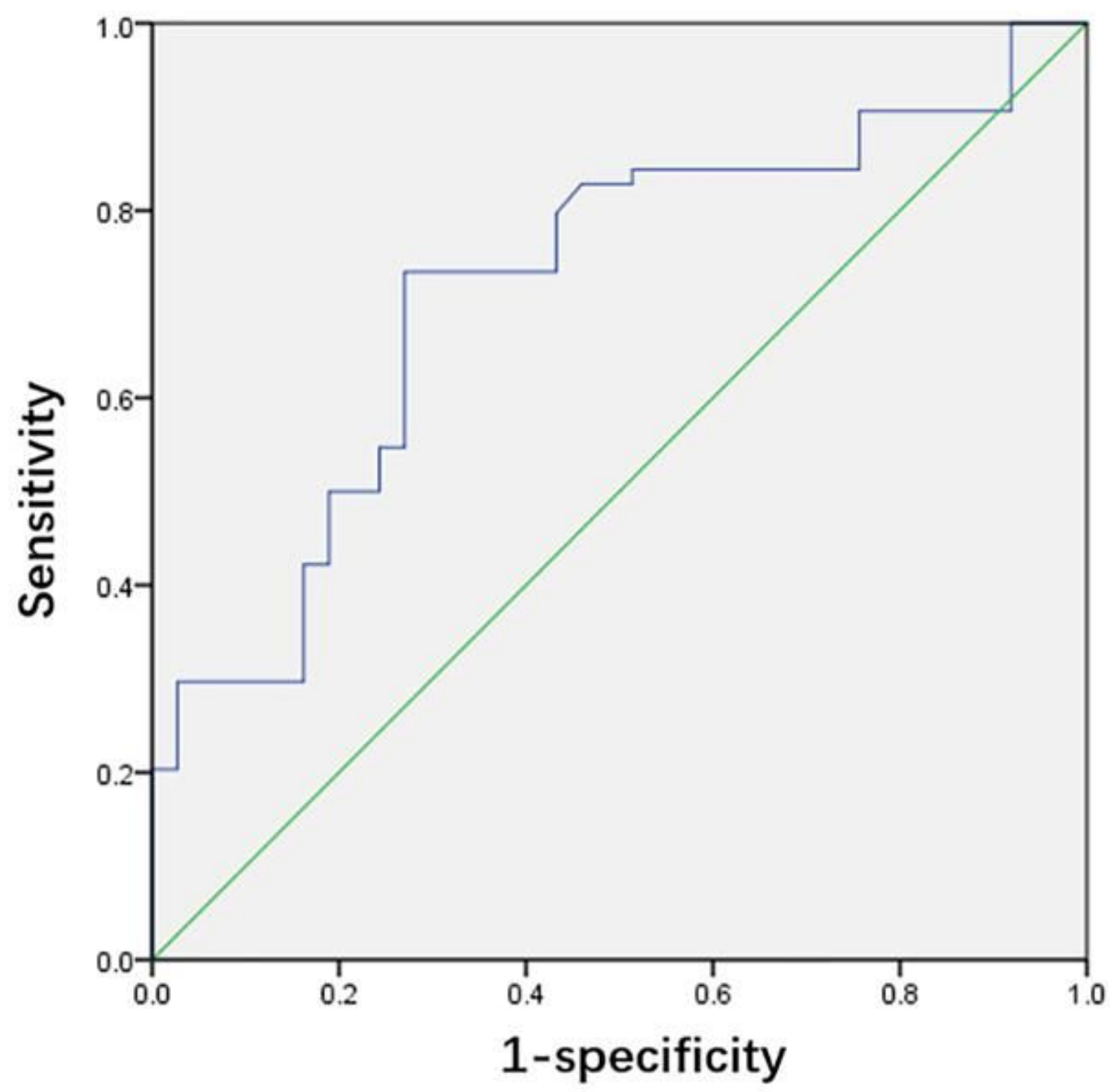

Figure 1

Receiver-operating characteristic (ROC) curve was constructed to determine the relationship between IVH and $\mathrm{VAH}$ in infarction volume. $\mathrm{AUC}=0.718(95 \% \mathrm{Cl}, 0.615-0.820)$, Youden index $(\mathrm{YI})=0.496, \mathrm{p}=0.002$ 
a

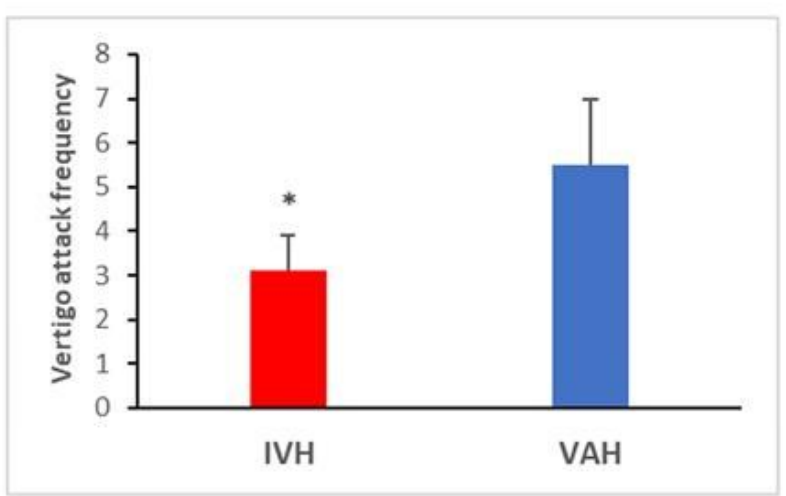

C

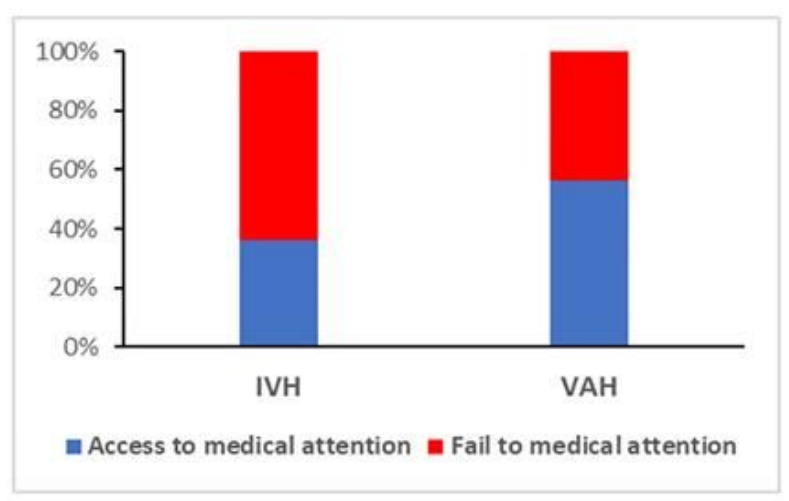

b

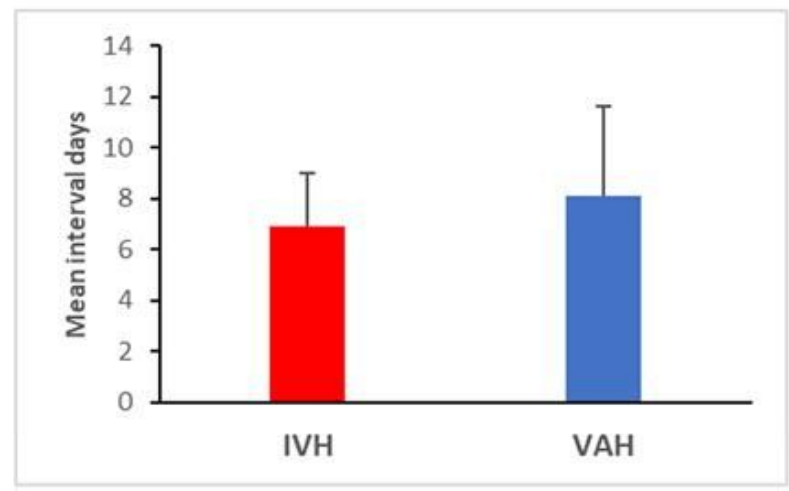

d

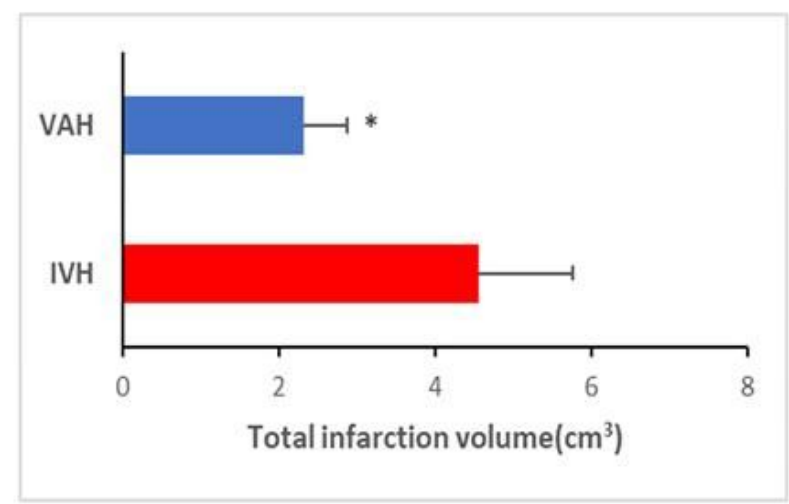

\section{Figure 2}

clinical characteristics comparison between IVH and AVH patients. (A) the episodes of vertigo symptom attack tended to be more frequent in AVH patients (median 3.1 vs. 5.5, $p<0 \cdot 03$ ); (B) the mean interval between the first vertigo symptom occurred and subsequent VB stroke showed no differences (median 6.9 vs. 8.1 days, $P=0.07)$; (C) less patients in IVH cohort sought medical intervention after vertigo arise ( $36.3 \%$ vs. $56.3 \%, p<0.02)$; (D) the total infarction volume in IVH patients tended to be larger than $\mathrm{AVH}$ group (4.56 cm3 vs. $2.32 \mathrm{~cm} 3, p=0.02$ ). 


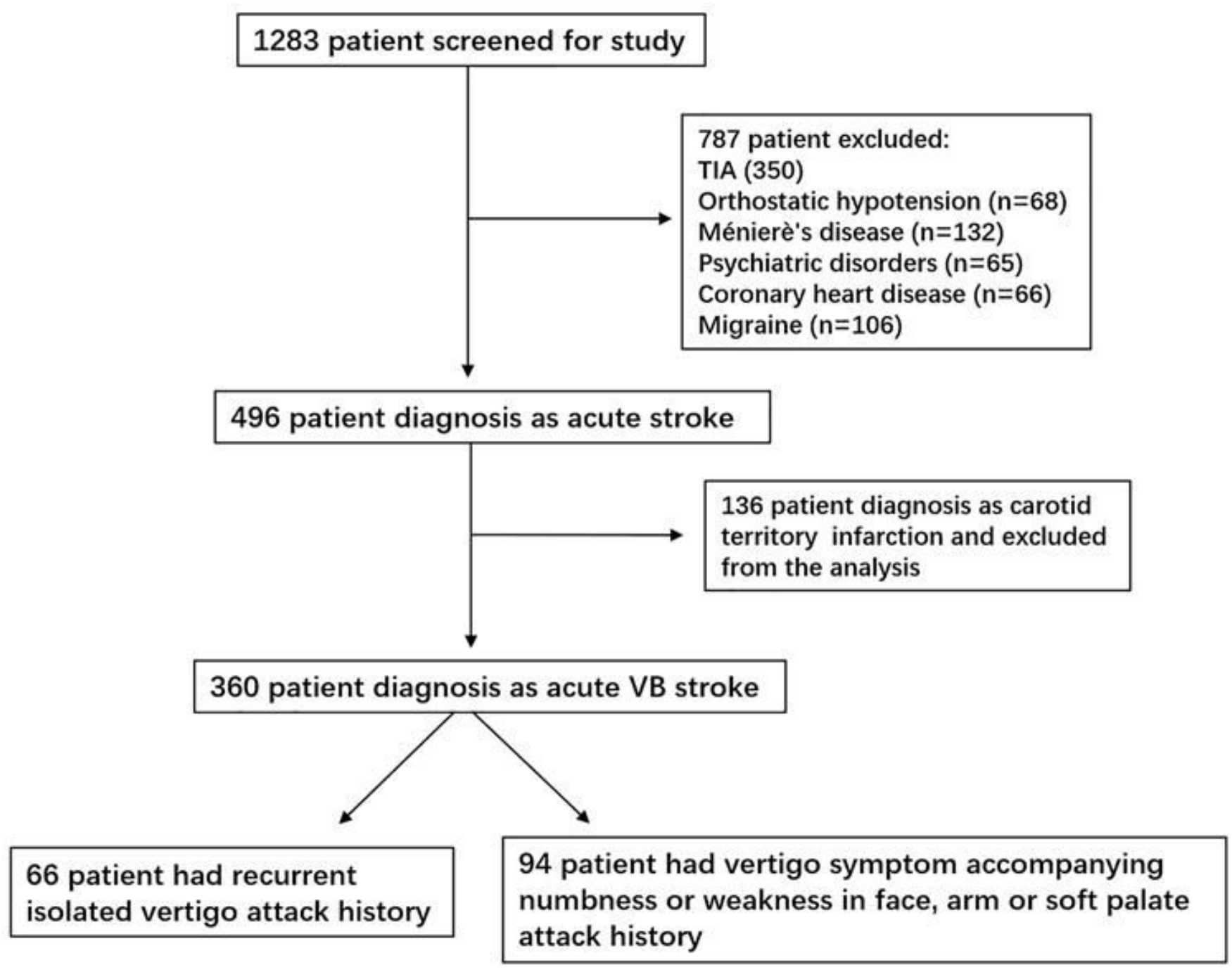

Figure 3

Study flow chart, TIA, transient ischemic attack, VB stroke: vertebrobasilar stroke 